\title{
No radiative generation of Chern-Simons-like term in Lorentz-violating QED: dealing with IR divergences
}

\author{
O.M. Del Cima, D.H.T. Franco and O. Piguet \\ Departamento de Física, Universidade Federal de Viçosa (UFV), \\ Campus Universitário, Avenida Peter Henry Rolfs s/n, 36570-900, Viçosa, MG, Brazil \\ E-mail: oswaldo.delcima@ufv.br, daniel.franco@ufv.br, \\ opiguet@pq.cnpq.br
}

ABSTRACT: The issue intensively claimed in the literature on the generation of a CPTodd and Lorentz violating Chern-Simons-like term by radiative corrections owing to a CPT violating interaction - the axial coupling of fermions with a constant vector field $b_{\mu}$ - is mistaken. The presence of massless gauge field triggers IR divergences that might show up from the UV subtractions, therefore, so as to deal with the (actual physical) IR divergences, the Lowenstein-Zimmermann subtraction scheme, in the framework of BPHZL renormalization method, has to be adopted. The proof on the non generation of such a Chern-Simons-like term is done, independent of any kind of regularization scheme, at all orders in perturbation theory.

Keywords: Anomalies in Field and String Theories, BRST Quantization, Renormalization Regularization and Renormalons, Space-Time Symmetries

ARXIV EPRINT: 1709.09756

In honor of Prof. Manfred Schweda

(1939-2017) 


\section{Contents}

1 Introduction 1

2 The model at the classical level 2

2.1 Continuous symmetries 3

$\begin{array}{lll}2.2 & \text { Discrete symmetries } & 6\end{array}$

3 The model at the quantum level $\quad 7$

3.1 The Lorentz-Ward and the Slavnov-Taylor identities: in search for anomalies 8

$\begin{array}{ll}3.2 \text { The stability condition: in search for counterterms } & 10\end{array}$

4 Conclusions

\section{Introduction}

Perturbative field models with symmetry breaking were investigated from the point of view of the theory of renormalization in the pioneering work of Symanzik [1, 2] and treated in a way that we can consider as definitive, by Becchi-Rouet-Stora [3-5]. However, several recent works, dealing in particular with field theories with Lorentz symmetry breaking, do not consider very carefully how the symmetry is broken, not taking into account the requirements that Symanzik-Becchi-Rouet-Stora have shown to be necessary. In this article, all our analysis will be based on a general iterative scheme called Algebraic Renormaliza$\operatorname{tion}^{1}[10-12]$. In the algebraic approach, in order to study the renormalizability of models characterized by a system of Ward identities, without referring to any special regularization procedure, two steps must be followed. In the first step, for a power-counting renormalizable model, at the level of the radiative corrections, one investigates the preservation of the symmetries, or the determination of all possible anomalies. This amounts to find the solution of the cohomology of its symmetry group: trivial elements (co-boundaries) correspond to breakings which can be compensated by non-invariant counterterms, whereas the non-trivial elements are the possible anomalies. These cohomology conditions are a generalization of the Wess-Zumino consistency condition [13] used in order to compute the possible anomalies of the Ward identities in Yang-Mills theories. In a second step, we check the stability of the classical action - which ensures that the quantum corrections do not produce counterterms corresponding to the renormalization of parameters not already present in the classical model.

Let us emphasize that the algebraic renormalization scheme is based on a set of general theorems of renormalization theory, collected under the name of Quantum Action Principle

\footnotetext{
${ }^{1}$ It should be emphasized that, based on the method suggested by the Epstein-Glaser construction [6-8], the algebraic method of renormalization was seeded by Stora in ref. [9].
} 
(QAP) [14-16]. These theorems deal with the whole of Feynman graphs combinatorics and integrability, so that explicit graph considerations are unnecessary - unless one looks for explicit quantitative results for applications to physics, of course. As Stora said: "Use the theorems!".

The quantum electrodynamics (QED) [17-20] with violation of Lorentz and CPT [2123] have been studied intensively. Among several issues, the possible generation of a ChernSimons-like term induced by radiative corrections arising from a CPT and Lorentz violating term in the fermionic sector has been a recurrent theme in the literature. We particularly mention the following works [24-44] (and references cited therein), where many controversies have emerged from the discussion whether this Chern-Simons-like term could be generated by means of radiative corrections arising from the axial coupling of charged fermions to a constant vector $b_{\mu}$ responsible for the breakdown of Lorentz symmetry.

In this work, we reassess the discussion on the radiative generation of a Chern-Simonslike term induced from quantum corrections in the extended QED. We show, to all orders in perturbation theory, that a CPT-odd and Lorentz violating Chern-Simons-like term, definitively, is not radiatively induced by the axial coupling of the fermions with the constant vector $b_{\mu}$. The proof of this fact is based on general theorems of perturbative quantum field theory (see [10-12] and references therein), where the Lowenstein-Zimmermann subtraction scheme in the framework of Bogoliubov-Parasiuk-Hepp-Zimmermann-Lowenstein (BPHZL) renormalization method [45] is adopted. The former has to be introduced, owing to the presence of massless gauge field, so as to subtract infrared (IR) divergences that should arise from the ultraviolet (UV) subtractions.

This article is structured as follows: in section 2, the quantum electrodynamics (QED) with a term which violates Lorentz and CPT (extended QED) is introduced, it is established all continuous and discrete simmetries at the classical level, as well as determined the ultraviolet and infrared dimensions of all the fields; the behaviour of the extended QED at the quantum level is analyzed in section 3; section 4 are left to the final comments and conclusions.

\section{The model at the classical level}

We start by considering an action for extended QED with a term which violates the Lorentz and CPT symmetries in the matter sector only. In the tree approximation, the classical action of extended QED with one Dirac spinor that we are considering here is given by:

$$
\Sigma^{(s-1)}=\Sigma_{\mathrm{S}}+\Sigma_{\mathrm{SB}}+\Sigma_{\mathrm{IR}}+\Sigma_{\mathrm{gf}}+\Sigma_{\mathrm{ext}}
$$

where

$$
\Sigma_{\mathrm{S}}=\int d^{4} x\left\{-\frac{1}{4} F^{\mu \nu} F_{\mu \nu}+i \bar{\psi} \gamma^{\mu} D_{\mu} \psi-m \bar{\psi} \psi\right\}
$$

is the symmetric part of $\Sigma^{(s-1)}$ under gauge and Lorentz transformations, and $D_{\mu} \psi \equiv$ $\left(\partial_{\mu}+i e A_{\mu}\right) \psi$. The term

$$
\Sigma_{\mathrm{SB}}=-\int d^{4} x b_{\mu} \bar{\psi} \gamma_{5} \gamma^{\mu} \psi
$$


is the non symmetric part of $\Sigma^{(s-1)}$. It violates CPT symmetry and breaks the manifest Lorentz covariance on account of a constant vector $b_{\mu}$, which selects a preferential direction in Minkowski space-time, breaking its isotropy. In addition to,

$$
\Sigma_{\mathrm{IR}}=\int d^{4} x \frac{1}{2} \mu^{2}(s-1) A_{\mu} A^{\mu},
$$

is the Lowenstein-Zimmermann mass term for the (massless) photon field. A LowensteinZimmermann mass term must be introduced in order to enable subtractions in momenta space without introducing spurious infrared (IR) singularities. The Lowenstein-Zimmermann parameter $s$ lies in the interval $0 \leq s \leq 1$ and plays the role of an additional subtraction variable (as the external momentum) in the BPHZL renormalization program, such that the theory describing a really massless particle is recovered for $s=1$. It should be comment that the Lowenstein-Zimmermann mass term for the photon field does not spoil gauge invariance at the quantum level $;^{2}$ this is a peculiarity of the abelian case [10]. Finally, in order to quantize the model, a gauge-fixing

$$
\Sigma_{\mathrm{gf}}=\int d^{4} x\left\{b \partial_{\mu} A^{\mu}+\frac{\xi}{2} b^{2}+\bar{c} \square c\right\}
$$

is added, together with the term, $\Sigma_{\text {ext }}$, by coupling the non-linear Becchi-Rouet-Stora (BRS) transformations to external sources

$$
\Sigma_{\text {ext }}=\int d^{4} x\{\bar{\Omega} \mathfrak{s} \psi-\mathfrak{s} \bar{\psi} \Omega\} .
$$

\subsection{Continuous symmetries}

The BRS transformations are given by:

$$
\begin{aligned}
\mathfrak{s} \psi & =i c \psi, & \mathfrak{s} \bar{\psi} & =-i c \bar{\psi} ; \\
\mathfrak{s} A_{\mu} & =-\frac{1}{e} \partial_{\mu} c, & \mathfrak{s} c & =0 ; \\
\mathfrak{s} \bar{c} & =\frac{1}{e} b, & \mathfrak{s} b & =0
\end{aligned}
$$

where $c$ is the ghost field, $\bar{c}$ is the antighost field and $b$ is the Lautrup-Nakanishi field [48, 49], respectively. Although not massive, the Faddeev-Popov ghosts, $c$ and $\bar{c}$, are free fields, they decouple, therefore, there is no need to introduce a Lowenstein-Zimmermann mass term for them.

The BRS invariance of the action is expressed in a functional way by the Slavnov-Taylor identity

$$
\mathcal{S}\left(\Sigma^{(s-1)}\right)=0
$$

where the Slavnov-Taylor operator $\mathcal{S}$ is defined, acting on an arbitrary functional $\mathcal{F}$, by

$$
\mathcal{S}(\mathcal{F})=\int d^{4} x\left\{-\frac{1}{e} \partial^{\mu} c \frac{\delta \mathcal{F}}{\delta A^{\mu}}+\frac{1}{e} b \frac{\delta \mathcal{F}}{\delta \bar{c}}+\frac{\delta \mathcal{F}}{\delta \bar{\Omega}} \frac{\delta \mathcal{F}}{\delta \psi}-\frac{\delta \mathcal{F}}{\delta \Omega} \frac{\delta \mathcal{F}}{\delta \bar{\psi}}\right\}
$$

\footnotetext{
${ }^{2}$ This was investigated in details for the QED in ref. $[46,47]$ using the BPHZ scheme.
} 
The corresponding linearized Slavnov-Taylor operator reads

$$
\mathcal{S}_{\mathcal{F}}=\int d^{4} x\left\{-\frac{1}{e} \partial^{\mu} c \frac{\delta}{\delta A^{\mu}}+\frac{1}{e} b \frac{\delta}{\delta \bar{c}}+\frac{\delta \mathcal{F}}{\delta \bar{\Omega}} \frac{\delta}{\delta \psi}+\frac{\delta \mathcal{F}}{\delta \psi} \frac{\delta}{\delta \bar{\Omega}}-\frac{\delta \mathcal{F}}{\delta \Omega} \frac{\delta}{\delta \bar{\psi}}-\frac{\delta \mathcal{F}}{\delta \bar{\psi}} \frac{\delta}{\delta \Omega}\right\} .
$$

The following nilpotency identities hold:

$$
\begin{aligned}
\mathcal{S}_{\mathcal{F}} \mathcal{S}(\mathcal{F}) & =0, & & \forall \mathcal{F}, \\
\mathcal{S}_{\mathcal{F}} \mathcal{S}_{\mathcal{F}} & =0 & & \text { if } \mathcal{S}(\mathcal{F})=0 .
\end{aligned}
$$

In particular, $\left(\mathcal{S}_{\Sigma}^{(s-1)}\right)^{2}=0$, since the action $\Sigma^{(s-1)}$ obeys the Slavnov-Taylor identity (2.8). The operation of $\mathcal{S}_{\Sigma^{(s-1)}}(2.10)$ upon the fields and the external sources reads

$$
\begin{aligned}
\mathcal{S}_{\Sigma^{(s-1)}} \phi & =s \phi, \quad \phi=\left\{\psi, \bar{\psi}, A_{\mu}, c, \bar{c}, b\right\}, \\
\mathcal{S}_{\Sigma^{(s-1)}} \Omega & =-\frac{\delta \Sigma^{(s-1)}}{\delta \bar{\psi}}, \\
\mathcal{S}_{\Sigma^{(s-1)}} \bar{\Omega}_{+} & =\frac{\delta \Sigma^{(s-1)}}{\delta \psi} .
\end{aligned}
$$

In addition to the Slavnov-Taylor identity (2.8), the classical action $\Sigma^{(s-1)}(2.1)$ is characterized by the gauge condition, the ghost equation and the antighost equation:

$$
\begin{aligned}
\frac{\delta \Sigma^{(s-1)}}{\delta b} & =\partial^{\mu} A_{\mu}+\xi b, \\
\frac{\delta \Sigma^{(s-1)}}{\delta \bar{c}} & =\square c, \\
-i \frac{\delta \Sigma^{(s-1)}}{\delta c} & =i \square \bar{c}+\bar{\Omega} \psi-\bar{\psi} \Omega .
\end{aligned}
$$

The action $\Sigma^{(s-1)}(2.1)$ is invariant also with respect to the rigid symmetry

$$
W_{\text {rigid }} \Sigma^{(s-1)}=0
$$

where the Ward operator, $W_{\text {rigid }}$, is defined by

$$
W_{\text {rigid }}=\int d^{4} x\left\{\psi \frac{\delta}{\delta \psi}-\bar{\psi} \frac{\delta}{\delta \bar{\psi}}+\Omega \frac{\delta}{\delta \Omega}-\bar{\Omega} \frac{\delta}{\delta \bar{\Omega}}\right\}
$$

On the other hand, the Lorentz symmetry is broken by the presence of the constant vector $b_{\mu}$. The fields $A_{\mu}$ and $\psi$ transform under infinitesimal Lorentz transformations $\delta x^{\mu}=\epsilon_{\nu}^{\mu} x^{\nu}\left(\epsilon_{\mu \nu}=-\epsilon_{\nu \mu}\right)$ - in such a way that

$$
\begin{aligned}
\delta_{\mathrm{L}} A_{\mu} & =-\epsilon^{\lambda}{ }_{\nu} x^{\nu} \partial_{\lambda} A_{\mu}+\epsilon_{\mu}{ }^{\nu} A_{\nu} \equiv \frac{1}{2} \epsilon^{\alpha \beta} \delta_{\mathrm{L} \alpha \beta} A_{\mu} ; \\
\delta_{\mathrm{L}} \psi & =-\epsilon^{\lambda}{ }_{\nu} x^{\nu} \partial_{\lambda} \psi-\frac{i}{4} \epsilon^{\mu \nu} \sigma_{\mu \nu} \psi \equiv \frac{1}{2} \epsilon^{\alpha \beta} \delta_{\mathrm{L} \alpha \beta} \psi, \\
\text { where } \sigma_{\mu \nu} & =\frac{i}{2}\left[\gamma_{\mu}, \gamma_{\nu}\right] .
\end{aligned}
$$


It has to be pointed out that, since the Lorentz breaking $\Sigma_{\mathrm{SB}}(2.3)$ is not linear in the quantum fields, it shall be submitted to renormalization. Nevertheless, it is a soft breaking, its ultraviolet (UV) power-counting dimension is less than 4, namely 3. A model with soft symmetry breakings is renormalizable if the radiative corrections do not induce a breakdown of the symmetries by terms of UV dimension equal to 4 , the hard breakings $[1,2]$. Bearing in mind the Weinberg's theorem [50], it can be concluded that the symmetry of the theory, in the asymptotic deep euclidean region of momentum space, remains preserved by radiative corrections. In order to control the Lorentz breaking and, in particular, its power-counting properties, by following $[1,2]$, and [51] for the specific case of Lorentz breaking, we introduce an external field $\beta_{\mu} \equiv \beta_{\mu}(x)$, with UV and IR dimensions equal to 1 , which transforms under Lorentz transformations as

$$
\delta_{\mathrm{L}} \beta_{\mu}=-\epsilon_{\nu}^{\lambda} x^{\nu} \partial_{\lambda} \beta_{\mu}+\epsilon_{\mu}{ }^{\nu}\left(\beta_{\nu}+b_{\nu}\right) \equiv \frac{1}{2} \epsilon^{\alpha \beta} \delta_{\mathrm{L} \alpha \beta} \beta_{\mu}
$$

The Ward operator associated to the Lorentz symmetry reads

$$
W_{\mathrm{L}}=\frac{1}{2} \epsilon^{\alpha \beta} W_{\mathrm{L} \alpha \beta},
$$

where

$$
W_{\mathrm{L} \alpha \beta}=\int d^{4} x \sum_{\varphi=A_{\mu}, \psi, \bar{\psi}, \beta_{\mu}} \delta_{\mathrm{L} \alpha \beta} \varphi \frac{\delta}{\delta \varphi} .
$$

By adding, to the action $\Sigma^{(s-1)}(2.1)$, a term depending on $\beta_{\mu}$, such as:

$$
\widetilde{\Sigma}^{(s-1)}=\Sigma^{(s-1)}-\int d^{4} x \beta_{\mu} \bar{\psi} \gamma_{5} \gamma^{\mu} \psi
$$

it can be verified the following classical Ward identity

$$
W_{\mathrm{L} \alpha \beta} \widetilde{\Sigma}^{(s-1)}=0
$$

so that, at $\beta_{\mu}=0$, it reduces to the broken Lorentz Ward identity

$$
W_{\mathrm{L} \alpha \beta} \Sigma^{(s-1)}=-b_{[\alpha} \int d^{4} x \bar{\psi} \gamma_{5} \gamma_{\beta]} \psi
$$

Owing to the fact that the external field $\beta_{\mu}$ is coupled (2.27) to the gauge invariant axial current $\left(j_{5}^{\mu}=\bar{\psi} \gamma_{5} \gamma^{\mu} \psi\right)$, it is assumed to be BRS invariant in order to preserve gauge invariance,

$$
\mathfrak{s} \int d^{4} x \beta_{\mu} \bar{\psi} \gamma_{5} \gamma^{\mu} \psi=0 \Longrightarrow \mathfrak{s} \beta_{\mu}=0
$$

Consequently, the action $\widetilde{\Sigma}^{(s-1)}(2.27)$ satisfies the same Slavnov identity (2.8) as the action $\Sigma^{(s-1)}(2.1)$ :

$$
\mathcal{S}\left(\widetilde{\Sigma}^{(s-1)}\right)=0,
$$


together with the following identities:

$$
\begin{aligned}
\frac{\delta \widetilde{\Sigma}^{(s-1)}}{\delta b} & =\partial^{\mu} A_{\mu}+\xi b, \\
\frac{\delta \widetilde{\Sigma}^{(s-1)}}{\delta \bar{c}} & =\square c, \\
-i \frac{\delta \widetilde{\Sigma}^{(s-1)}}{\delta c} & =i \square \bar{c}+\bar{\Omega} \psi-\bar{\psi} \Omega, \\
W_{\text {rigid }} \widetilde{\Sigma}^{(s-1)} & =0 .
\end{aligned}
$$

\subsection{Discrete symmetries}

Charge conjugation. Assuming the Dirac representation of the $\gamma$-matrices [52], the charge conjugation transformations read:

$$
\begin{gathered}
\psi \stackrel{\mathrm{C}}{\longrightarrow} C \bar{\psi}^{T}=i \gamma^{2} \gamma^{0} \bar{\psi}^{T}, \\
A_{\mu} \stackrel{\mathrm{C}}{\longrightarrow}-A_{\mu}, \\
C \gamma_{\mu} C^{-1}=-\gamma_{\mu}^{T},
\end{gathered}
$$

then, it is verified that all terms in the action $\widetilde{\Sigma}^{(s-1)}(2.27)$ are invariant under charge conjugation.

Parity. The parity transformations are given by:

$$
\begin{aligned}
x^{\mu} & \stackrel{\mathrm{P}}{\longrightarrow} x_{\mu}, \\
\psi & \stackrel{\mathrm{P}}{\longrightarrow} P \psi=\gamma^{0} \psi, \\
A^{\mu} & \stackrel{\mathrm{P}}{\longrightarrow} A_{\mu}, \\
P \gamma_{\mu} P^{-1} & =-(-1)^{\eta_{\mu \mu}} \gamma_{\mu},
\end{aligned}
$$

where in this case all terms of the action $\widetilde{\Sigma}^{(s-1)}(2.27)$, except the Lorentz breaking term $\Sigma_{\mathrm{SB}}(2.3)$, are invariant under parity.

Time reversal. The time reversal transformations follow:

$$
\begin{gathered}
x^{\mu} \stackrel{\mathrm{T}}{\longrightarrow}-x_{\mu}, \\
\psi \stackrel{\mathrm{T}}{\longrightarrow} T \psi=i \gamma^{1} \gamma^{3} \psi, \\
A^{\mu} \stackrel{\mathrm{T}}{\longrightarrow} A_{\mu}, \\
T \gamma^{\mu} T^{-1}=\gamma_{\mu}^{T},
\end{gathered}
$$

where it is verified that the Lorentz breaking term $\Sigma_{\mathrm{SB}}(2.3)$ is not invariant under time reversal, whereas the other terms in the action $\widetilde{\Sigma}^{(s-1)}(2.27)$ remain invariant.

Consequently, the action $\widetilde{\Sigma}^{(s-1)}(2.27)$, has CPT symmetry broken by the Lorentz breaking term, $\Sigma_{\mathrm{SB}}(2.3)$ :

$$
\bar{\psi} b_{\mu} \gamma_{5} \gamma^{\mu} \psi \stackrel{\mathrm{CPT}}{\longrightarrow}-\bar{\psi} b_{\mu} \gamma_{5} \gamma^{\mu} \psi
$$




\begin{tabular}{|c|c|c|c|c|c|c|c|c|c|}
\hline & $A_{\mu}$ & $\psi$ & $c$ & $\bar{c}$ & $b$ & $\Omega$ & $\beta_{\mu}$ & $s-1$ & $s$ \\
\hline$d$ & 1 & $3 / 2$ & 0 & 2 & 2 & $5 / 2$ & 1 & 1 & 1 \\
\hline$r$ & 1 & 2 & 0 & 2 & 2 & 2 & 1 & 1 & 0 \\
\hline$\Phi \Pi$ & 0 & 0 & 1 & -1 & 0 & -1 & 0 & 0 & 0 \\
\hline$G P$ & 0 & 1 & 1 & 1 & 0 & 0 & 0 & 0 & 0 \\
\hline
\end{tabular}

Table 1. UV $(d)$ and IR $(r)$ dimensions, ghost number $(\Phi \Pi)$ and Grassmann parity $(G P)$.

UV and IR dimensions. Switching off the coupling constant $(e)$ and taking the free part of the action (2.1), the tree-level propagators in momenta space, for all the fields, read:

$$
\begin{aligned}
\Delta_{\bar{\psi} \psi}(k) & =i \frac{\not k+m}{k^{2}-m^{2}} \\
\Delta_{A A}^{\mu \nu}(k, s) & =-i\left\{\frac{1}{k^{2}-M^{2}(s-1)^{2}}\left(\eta^{\mu \nu}-\frac{k^{\mu} k^{\nu}}{k^{2}}\right)+\frac{\xi}{k^{2}-\xi M^{2}(s-1)^{2}} \frac{k^{\mu} k^{\nu}}{k^{2}}\right\}, \\
\Delta_{A b}^{\mu}(k) & =\frac{k^{\mu}}{k^{2}}, \quad \Delta_{b b}(k)=0 \\
\Delta_{\bar{c} c}(k) & =-i \frac{1}{k^{2}} .
\end{aligned}
$$

The ultraviolet (UV) and infrared (IR) dimensions of any fields, $X$ and $Y$, are given by the UV and IR asymptotic behaviour of their propagator $\left(\Delta_{X Y}(k, s)\right), d_{X Y}$ and $r_{X Y}$, respectively, defined as follows:

$$
\begin{aligned}
& d_{X Y}=\overline{\operatorname{deg}}_{(k, s)} \Delta_{X Y}(k, s), \\
& r_{X Y}=\underline{\operatorname{deg}}(k, s-1) \\
& \Delta_{X Y}(k, s),
\end{aligned}
$$

where the upper degree $\overline{\operatorname{deg}}_{(k, s)}$ gives the asymptotic power for $(k, s) \rightarrow \infty$ whereas the lower degree $\underline{\mathrm{deg}}(k, s-1)$ gives the asymptotic power for $(k, s-1) \rightarrow 0$. The UV $(d)$ and IR $(r)$ dimensions of the fields, $X$ and $Y$, shall respect the following inequalities:

$$
d_{X}+d_{Y} \geqslant 4+d_{X Y} \text { and } r_{X}+r_{Y} \leqslant 4+r_{X Y} .
$$

In summary, the UV $(d)$ and IR $(r)$ dimensions - which are those involved in the Lowenstein-Zimmermann subtraction scheme [45] — as well as the ghost numbers $(\Phi \Pi)$ and the Grassmann parity (GP) of all fields are displayed in table 1. It should be stressed that the statistics among the fields is defined as follows: the integer spin fields with odd ghost number, as well as, the half integer spin fields with even ghost number anticommute among themselves. However, the other fields commute with the formers and also among themselves.

\section{The model at the quantum level}

Following Symanzik — "whether you like it or not, you have to include in the lagrangian all counter terms consistent with locality and power-counting, unless otherwise constrained 
by Ward identities" [53] — we present next, the perturbative quantization of the extended QED model, using the algebraic renormalization method [11, 12]. Our aim is to prove that the full quantum model has the same properties as the classical model, namely, we have to demonstrate that, at the quantum level, the Ward identity related to the Lorentz symmetry (2.28) and the Slavnov-Taylor identity associated to the gauge symmetry (2.31) are satisfied at all orders in perturbation theory:

$$
\begin{gathered}
\left.W_{\mathrm{L} \alpha \beta} \Gamma^{(s-1)}\right|_{s=1}=0, \\
\left.\mathcal{S}\left(\Gamma^{(s-1)}\right)\right|_{s=1}=0 .
\end{gathered}
$$

In order to study the renormalizability of models characterized by a system of Ward identities, without referring to any special regularization scheme, two procedures must be followed $[11,12]$. First, we search for possible anomalies of the Ward identities through an analysis of the Wess-Zumino consistency condition. Second, we verify the stability of the classical action, which guarantees that the quantum corrections do not produce counterterms corresponding to the renormalization of parameters which are not already present at the classical level.

\subsection{The Lorentz-Ward and the Slavnov-Taylor identities: in search for anoma- lies}

At the quantum level the vertex functional, $\Gamma^{(s-1)}$, which coincides with the classical action, $\widetilde{\Sigma}^{(s-1)}(2.27)$, at zeroth order in $\hbar$,

$$
\Gamma^{(s-1)}=\widetilde{\Sigma}^{(s-1)}+\mathcal{O}(\hbar),
$$

has to satisfy the same constraints as the classical action, namely eq. (2.28) and eqs. (2.31)-(2.35).

According to the Quantum Action Principle [14-16], due to radiative corrections, the Lorentz symmetry Ward identity (2.28) and the Slavnov-Taylor identity (2.31) develop quantum breakings:

$$
\begin{aligned}
\left.W_{\mathrm{L} \alpha \beta} \Gamma^{(s-1)}\right|_{s=1} & =\left.\Delta_{\mathrm{L} \alpha \beta} \cdot \Gamma^{(s-1)}\right|_{s=1} \\
& =\Delta_{\mathrm{L} \alpha \beta}+\mathcal{O}\left(\hbar \Delta_{\mathrm{L} \alpha \beta}\right), \\
\left.\mathcal{S}\left(\Gamma^{(s-1)}\right)\right|_{s=1} & =\left.\Delta \cdot \Gamma^{(s-1)}\right|_{s=1} \\
& =\Delta_{\mathrm{g}}+\mathcal{O}\left(\hbar \Delta_{\mathrm{g}}\right),
\end{aligned}
$$

where $\left.\Delta_{\mathrm{L} \alpha \beta} \equiv \Delta_{\mathrm{L} \alpha \beta}\right|_{s=1}$ and $\left.\Delta_{\mathrm{g}} \equiv \Delta_{\mathrm{g}}\right|_{s=1}$ are integrated local functionals, taken at $s=1$, with ghost number one and, UV and IR dimensions bounded by $\delta \leq 4$ and $\rho \geq 4$, respectively.

The validity of the Lorentz Ward identity has been proved in [51] by using the Whitehead's lemma for semi-simple Lie groups, which states the vanishing of the first cohomology of such kind of group $[3,5]$. Here ${ }^{3}$ this means that the Lorentz symmetry breaking $\Delta_{\mathrm{L} \alpha \beta}(3.4)$, can be written as

$$
\Delta_{\mathrm{L} \alpha \beta}=W_{\mathrm{L} \alpha \beta} \widehat{\Delta}_{\mathrm{L}}
$$

\footnotetext{
${ }^{3}$ See details in [42].
} 
where $\widehat{\Delta}_{\mathrm{L}}$ is an integrated local insertion of UV and IR dimensions bounded by $\delta \leq 4$ and $\rho \geq 4$, respectively. Therefore, $\widehat{\Delta}_{\mathrm{L}}$ can be reabsorbed in the action as a noninvariant counterterm, order by order, establishing the Lorentz Ward identity (3.1) at the quantum level.

The nilpotency identity (2.11) together with

$$
\mathcal{S}_{\Gamma^{(s-1)}}=\mathcal{S}_{\widetilde{\Sigma}^{(s-1)}}+\mathcal{O}(\hbar)
$$

implies the following consistency condition for the gauge symmetry breaking $\Delta_{\mathrm{g}}(3.5)$ :

$$
\mathcal{S}_{\widetilde{\Sigma}^{(s-1)}} \Delta_{\mathrm{g}}=0
$$

and beyond that, $\Delta_{\mathrm{g}}$ also satisfy the constraints:

$$
\frac{\delta \Delta_{\mathrm{g}}}{\delta b}=\frac{\delta \Delta_{\mathrm{g}}}{\delta \bar{c}}=\int d^{4} x \frac{\delta \Delta_{\mathrm{g}}}{\delta c}=W_{\text {rigid }} \Delta_{\mathrm{g}}=W_{\mathrm{L} \alpha \beta} \Delta_{\mathrm{g}}=0 .
$$

The Wess-Zumino consistency condition (3.8) constitutes a cohomology problem in the sector of ghost number one. Its solution can always be written as a sum of a trivial cocycle $\mathcal{S}_{\Sigma^{(s-1)}} \widehat{\Delta}_{\mathrm{g}}^{(0)}$, where $\widehat{\Delta}_{\mathrm{g}}^{(0)}$ has ghost number zero, and of nontrivial elements belonging to the cohomology of $\mathcal{S}_{\widetilde{\Sigma}^{(s-1)}}(2.10)$ in the sector of ghost number one:

$$
\Delta_{\mathrm{g}}^{(1)}=\widehat{\Delta}_{\mathrm{g}}^{(1)}+\mathcal{S}_{\widetilde{\Sigma}^{(s-1)}} \widehat{\Delta}_{\mathrm{g}}^{(0)} .
$$

However, considering the Slavnov-Taylor operator $\mathcal{S}_{\widetilde{\Sigma}^{(s-1)}}(2.10)$ and the quantum breaking (3.5), it results that $\Delta_{\mathrm{g}}^{(1)}$ exhibits UV and IR dimensions bounded by $\delta \leq 4$ and $\rho \geq 4$.

From the antighost equation in (3.9):

$$
\int d^{4} x \frac{\delta \widehat{\Delta}_{\mathrm{g}}^{(1)}}{\delta c}=0,
$$

it follows that $\widehat{\Delta}_{\mathrm{g}}^{(1)}$ can be written as

$$
\widehat{\Delta}_{\mathrm{g}}^{(1)}=\int d^{4} x \mathcal{T}_{\mu} \partial^{\mu} c
$$

where $\mathcal{T}_{\mu}$ is a rank- 1 tensor with ghost number zero, with UV and IR dimensions bounded by $d \leq 3$ and $r \geq 3$, respectively. However, the tensor $\mathcal{T}_{\mu}$ can be split into two pieces:

$$
\mathcal{T}_{\mu}=r_{\mathrm{v}} \mathcal{V}_{\mu}+r_{\mathrm{p}} \mathcal{P}_{\mu}
$$

where $\mathcal{V}_{\mu}$ is a vector and $\mathcal{P}_{\mu}$ is a pseudo-vector, with $r_{\mathrm{v}}$ and $r_{\mathrm{p}}$ being coefficients to be determined. By considering the UV and IR dimensional constraints to be satisfied by $\mathcal{T}_{\mu}$ (3.13) together with the conditions upon the Slavnov-Taylor breaking $\widehat{\Delta}_{\mathrm{g}}^{(1)}$, given by (3.8) and (3.9), it follows that:

$$
\mathcal{T}_{\mu}=r_{\mathrm{v}} \partial^{\rho} F_{\rho \mu}+r_{\mathrm{p}} \epsilon_{\mu \nu \rho \sigma} A^{\nu} F^{\rho \sigma}
$$


Consequently, substituting (3.14) into (3.12), the gauge symmetry breaking $\widehat{\Delta}_{\mathrm{g}}^{(1)}$ reads:

$$
\widehat{\Delta}_{\mathrm{g}}^{(1)}=-\frac{r_{\mathrm{p}}}{2} \int d^{4} x c \epsilon_{\mu \nu \rho \sigma} F^{\mu \nu} F^{\rho \sigma},
$$

which is the well-known (Adler-Bardeen-Bell-Jackiw) ABBJ-anomaly [54-56]. Therefore, up to noninvariant counterterms, which are $\mathcal{S}_{\widetilde{\Sigma}^{(s-1)}}$-variations of the integrated local insertions $\widehat{\Delta}_{\mathrm{g}}^{(0)}$ :

$$
\Delta_{\mathrm{g}}^{(1)}=\mathcal{S}_{\widetilde{\Sigma}^{(s-1)}} \widehat{\Delta}_{\mathrm{g}}^{(0)}-\frac{r_{\mathrm{p}}}{2} \int d^{4} x c \epsilon_{\mu \nu \rho \sigma} F^{\mu \nu} F^{\rho \sigma} .
$$

The anomaly coefficient $r_{\mathrm{p}}$ does not get renormalizations [11, 57-59], besides that, if it vanishes at the one loop order, it is in fact identically zero, thus it is enough to check its vanishing at that order. However, owing to the fact that the potentially dangerous axial current $j_{5}^{\mu}=\bar{\psi} \gamma_{5} \gamma^{\mu} \psi$ is coupled only to the external (classical) field $\beta_{\mu}$ - and not to any quantum field of the model - there is no gauge anomaly stemming from $[10,33$, 34]. Consequently, it follows that the Slavnov-Taylor identity (3.2) is accomplished at the quantum level.

Concerning the potential anomalies, it can be concluded that the presence of the CPT violating interaction term $\Sigma_{\mathrm{SB}}(2.3)$, which couples the axial fermion current $j_{5}^{\mu}=$ $\bar{\psi} \gamma_{5} \gamma^{\mu} \psi$ to a constant vector field $b_{\mu}$, does not induce at any order in perturbation theory, independent of any regularization scheme, neither a Lorentz anomaly nor a gauge anomaly.

\subsection{The stability condition: in search for counterterms}

In order to verify if the action in the tree-approximation $\left(\widetilde{\Sigma}^{(s-1)}\right)$ is stable under radiative corrections, we perturb it by an arbitrary integrated local functional (counterterm) $\widetilde{\Sigma}^{c(s-1)}$, such that

$$
\widehat{\Sigma}^{(s-1)}=\widetilde{\Sigma}^{(s-1)}+\varepsilon \widetilde{\Sigma}^{c(s-1)},
$$

where $\varepsilon$ is an infinitesimal parameter. The functional $\left.\widetilde{\Sigma}^{c} \equiv \widetilde{\Sigma}^{c(s-1)}\right|_{s=1}$ has the same quantum numbers as the action in the tree-approximation at $s=1$.

The deformed action $\widehat{\Sigma}^{(s-1)}$ must still obey all the conditions presented above, henceforth, $\widetilde{\Sigma}^{c(s-1)}$ is subjected to the following set of constraints:

$$
\begin{aligned}
\mathcal{S}_{\Sigma^{(s-1)}} \widetilde{\Sigma}^{c(s-1)} & =0, \\
\frac{\delta \widetilde{\Sigma}^{c(s-1)}}{\delta b} & =\frac{\delta \widetilde{\Sigma}^{c(s-1)}}{\delta \bar{c}}=\frac{\delta \widetilde{\Sigma}^{c(s-1)}}{\delta c}=0, \\
W_{\text {rigid }} \widetilde{\Sigma}^{c(s-1)} & =0, \\
W_{\mathrm{L} \alpha \beta} \widetilde{\Sigma}^{c(s-1)} & =0 .
\end{aligned}
$$

The most general invariant counterterm $\widetilde{\Sigma}^{c(s-1)}$ — the most general field polynomial — with UV and IR dimensions bounded by $\delta \leq 4$ and $\rho \geq 4$, with ghost number zero and fulfilling the conditions displayed in eqs. (3.18)-(3.21), reads:

$$
\left.\widetilde{\Sigma}^{c(s-1)}\right|_{\rho \geq 4} ^{\delta \leq 4}=\int d^{4} x\left\{\alpha_{1} i \bar{\psi} \gamma^{\mu}\left(\partial_{\mu}+i e A_{\mu}\right) \psi+\alpha_{2} \bar{\psi} \psi+\alpha_{3} F^{\mu \nu} F_{\mu \nu}+\alpha_{4}\left(\beta_{\mu}+b_{\mu}\right) \bar{\psi} \gamma_{5} \gamma^{\mu} \psi\right\} .
$$


The coefficients $\alpha_{1}, \ldots, \alpha_{4}$ are arbitrary, and they are fixed, order by order in perturbation theory, by the following four normalization conditions:

$$
\begin{aligned}
\left.\Gamma_{\bar{\psi} \psi}(\not p)\right|_{\not p=m} & =0,\left.\quad \frac{\partial}{\partial \not p} \Gamma_{\bar{\psi} \psi}(\not p)\right|_{\not p=m}=1, \\
\left.\frac{\partial}{\partial p^{2}} \Gamma_{A_{\mu} A_{\mu}}\left(p^{2}\right)\right|_{p^{2}=\kappa^{2}} ^{s=1} & =1, \\
-\left.\frac{1}{4} \operatorname{Tr}\left[\gamma^{\mu} \gamma^{5} \Gamma_{\beta_{\mu} \bar{\psi} \psi}(0, \not p)\right]\right|_{\not p=m} & =1 .
\end{aligned}
$$

Notwithstanding, it shall be stressed here that, a Chern-Simons-like term of the type

$$
\left.\Sigma_{\mathrm{CS}}\right|_{\rho \geq 4} ^{\delta \leq 4}=\int d^{4} x \alpha_{5}\left\{\left.\epsilon_{\mu \nu \alpha \beta} \beta^{\mu} A^{\nu} \partial^{\alpha} A^{\beta}\right|_{4} ^{4}+\left.\epsilon_{\mu \nu \alpha \beta} b^{\mu} A^{\nu} \partial^{\alpha} A^{\beta}\right|_{3} ^{3}\right\}
$$

in spite of fulfils the conditions (3.19)-(3.21), its first term breaks gauge invariance by violating the Slavnov-Taylor identity (3.18), whereas its second term violates the IR dimension constraint $(\rho \geq 4)$, it has IR dimension equal to three. Therefore, the Chern-Simons-like term $\Sigma_{\mathrm{CS}}(3.24)$ can never be generated by radiative corrections if the renormalization procedure is performed correctly. First, by taking care of the IR divergences - for instance, through the Lowenstein-Zimmermann method [45] - that show up, thanks to the presence of the photon, which is massless. Second, by properly treating and controlling the Lorentz symmetry breaking through the Symanzik method [1,2]. Anyway, even though the external field $\beta_{\mu}$ was not introduced in order to control the Lorentz breaking, the Chern-Simons-like term — which is a soft Lorentz breaking (UV dimension less than four) — would not be radiatively generated as explained above, nevertheless, any gauge invariant hard Lorentz breaking (UV dimension equal to four) could be induced by radiative corrections. In summary, a CPT-odd and Lorentz-violating Chern-Simons-like term is definitely not radiatively induced at any order in perturbation theory, independent of any regularization scheme, by coupling the axial fermion current $j_{5}^{\mu}=\bar{\psi} \gamma_{5} \gamma^{\mu} \psi$ to a constant vector field $b_{\mu}$.

\section{Conclusions}

In this work we reassess the discussion on the radiative generation of a Chern-Simons-like term induced from quantum corrections in the extended QED. We prove, to all orders in perturbation theory, that a CPT-odd and Lorentz violating Chern-Simons-like term, definitively, is not radiatively induced by the axial coupling of the fermions with the constant vector $b_{\mu}$. The proof of this fact is based on general theorems of perturbative quantum field theory, where the Lowenstein-Zimmermann subtraction scheme in the framework of Bogoliubov-Parasiuk-Hepp-Zimmermann-Lowenstein (BPHZL) renormalization method is adopted.

It is true that we need new ideas to go beyond the Standard Model, for instance, which is the case of the Lorentz symmetry breaking, where if it is manifested or not in our universe has been the subject of much discussion, however so far, no trace was found up to now. 
Experiments are the final judgement of a theory, which has to be checked experimentally, but the Lorentz symmetry breaking still remains a theoretical construction, regardless of how seductive the idea can be. Nevertheless, even as a theoretical construction, the idea of the Lorentz symmetry breaking should be well grounded and treated properly, although it seems that is not the case in the recent literature on the subject.

Particularly here, we analyze the issue intensively studied in recent years on the generation of a Lorentz violating Chern-Simons-like term by radiative corrections in the extended QED. Unfortunately, several recent works, dealing on the subject, do not consider very carefully the Lorentz symmetry breaking - neither at the classical level nor at the quantum level — not taking into account the requirements that Symanzik-Becchi-Rouet-Stora have shown to be necessary. Those authors should read the seminal works by SymanzikBecchi-Rouet-Stora [1-5] and devour them.

It shall be stressed that it is urgent and mandatory the reconsideration of the fundamental works on renormalization of quantum field models developed mainly in the 1970's, especially the articles by Symanzik-Becchi-Rouet-Stora on renormalizable models with broken symmetry, which provides an appropriate theoretical tool susceptible to avoid some bad conclusions associated with models with broken Lorentz symmetry. It is important to emphasize that, the main characteristic of this method is the control of the breaking and, in particular, its power-counting properties, converting the initial action containing terms that violate the Lorentz symmetry into one which is invariant under the original transformation by adding external fields (the Symanzik sources). Without this control, the study of the stability (here meant additive renormalization) tells us that any term that breaks the Lorentz symmetry, compatible with the power-counting, must necessarily be present in the starting (classical) action. On the other hand, if we include in the initial (classical) action all terms that break the Lorentz symmetry, compatible with the locality and power-counting, no breaking control is required (see ref. [43]). Therefore, paraphrasing Symanzik, whether you like it or not, you have to include in the classical action all Lorentz violating terms consistent with locality and power-counting, unless otherwise constrained by a breaking control.

\section{Acknowledgments}

The authors dedicate this work to the memory of Prof. Manfred Schweda (1939-2017). O.M.D.C. dedicates this work to his father (Oswaldo Del Cima, in memoriam), mother (Victoria M. Del Cima, in memoriam), daughter (Vittoria) and son (Enzo). This work was partially funded by FAPEMIG and CNPq (O.P.).

Open Access. This article is distributed under the terms of the Creative Commons Attribution License (CC-BY 4.0), which permits any use, distribution and reproduction in any medium, provided the original author(s) and source are credited. 


\section{References}

[1] K. Symanzik, Renormalizable models with simple symmetry breaking. I. Symmetry breaking by a source term, Commun. Math. Phys. 16 (1970) 48 [InSPIRE].

[2] K. Symanzik, Renormalization of theories with broken symmetry, in Cargèse Lectures in Physics, Vol. 5, D. Bessis ed., Gordon \& Breach (1971).

[3] R. Stora, Renormalizable models with broken symmetries, in Renormalization theory, G. Velo and A.S. Wightman eds., D. Reidel Publ. Co. (1976).

[4] J.H. Lowenstein, A. Rouet, R. Stora and W. Zimmermann, Renormalizable models with broken symmetries, in Renormalization and invariance in quantum field theory, E.R. Caianiello ed., Plenum Press (1981).

[5] C. Becchi, A. Rouet and R. Stora, Renormalizable theories with symmetry breaking, in Field theory, quantization and statistical physics, E. Tirapegui ed., D. Reidel Publ. Co. (1981).

[6] H. Epstein and V. Glaser, The role of locality in perturbation theory, Ann. Inst. Henri Poincaré A 19 (1973) 211.

[7] W. Zimmermann, Composite operators in the perturbation theory of renormalizable interactions, Annals Phys. 77 (1973) 536 [INSPIRE].

[8] W. Zimmermann, Normal products and the short distance expansion in the perturbation theory of renormalizable interactions, Annals Phys. 77 (1973) 570 [INSPIRE].

[9] R. Stora, Lagrangian field theory, in Particle physics, proceedings of the Les Houches Summer School (1971), C. De Witt and C. Itzykson eds., Gordon \& Breach (1973).

[10] O. Piguet and A. Rouet, Symmetries in perturbative quantum field theory, Phys. Rept. 76 (1981) 1 [inSPIRE].

[11] O. Piguet and S.P. Sorella, Algebraic renormalization: perturbative renormalization, symmetries and anomalies, Lecture Notes in Physics Monographs, Vol. 28, Springer (1995).

[12] A. Boresch et al., Applications of noncovariant gauges in the algebraic renormalization procedure, World Scientific (1998).

[13] J. Wess and B. Zumino, A Lagrangian model invariant under supergauge transformations, Phys. Lett. B 49 (1974) 52 [inSPIRE].

[14] Y.-M.P. Lam, Perturbation Lagrangian theory for scalar fields: Ward-Takahasi identity and current algebra, Phys. Rev. D 6 (1972) 2145 [InSPIRE].

[15] J.H. Lowenstein, Differential vertex operations in Lagrangian field theory, Commun. Math. Phys. 24 (1971) 1 [InSPIRE].

[16] F. Brenneke and M. Dütsch, The quantum action principle in the framework of causal perturbation theory, in Quantum field theory: competitive models, B. Fauser, J. Tolksdorf and E. Zeidler eds., Birkhäuser (2009).

[17] S.M. Carroll, G.B. Field and R. Jackiw, Limits on a Lorentz and parity violating modification of electrodynamics, Phys. Rev. D 41 (1990) 1231 [INSPIRE].

[18] D. Colladay and V.A. Kostelecký, CPT violation and the standard model, Phys. Rev. D 55 (1997) 6760 [hep-ph/9703464] [INSPIRE].

[19] D. Colladay and V.A. Kostelecký, Lorentz violating extension of the standard model, Phys. Rev. D 58 (1998) 116002 [hep-ph/9809521] [InSPIRE].

[20] V.A. Kostelecký, C.D. Lane and A.G.M. Pickering, One loop renormalization of Lorentz violating electrodynamics, Phys. Rev. D 65 (2002) 056006 [hep-th/0111123] [InSPIRE]. 
[21] O.W. Greenberg, CPT violation implies violation of Lorentz invariance, Phys. Rev. Lett. 89 (2002) 231602 [hep-ph/0201258] [INSPIRE].

[22] M. Chaichian, K. Fujikawa and A. Tureanu, Lorentz invariant CPT violation, Eur. Phys. J. C 73 (2013) 2349 [arXiv: 1205.0152] [InSPIRE].

[23] M. Chaichian, K. Fujikawa and A. Tureanu, Electromagnetic interaction in theory with Lorentz invariant CPT violation, Phys. Lett. B 718 (2013) 1500 [arXiv:1210.0208] [INSPIRE].

[24] S.R. Coleman and S.L. Glashow, High-energy tests of Lorentz invariance, Phys. Rev. D 59 (1999) 116008 [hep-ph/9812418] [INSPIRE].

[25] R. Jackiw and V.A. Kostelecký, Radiatively induced Lorentz and CPT violation in electrodynamics, Phys. Rev. Lett. 82 (1999) 3572 [hep-ph/9901358] [INSPIRE].

[26] J.M. Chung, Lorentz and CPT violating Chern-Simons term in the formulation of functional integral, Phys. Rev. D 60 (1999) 127901 [hep-th/9904037] [INSPIRE].

[27] J.M. Chung, Radiatively induced Lorentz and CPT violating Chern-Simons term in QED, Phys. Lett. B 461 (1999) 138 [hep-th/9905095] [INSPIRE].

[28] M. Pérez-Victoria, Exact calculation of the radiatively induced Lorentz and CPT violation in QED, Phys. Rev. Lett. 83 (1999) 2518 [hep-th/9905061] [INSPIRE].

[29] W.F. Chen, Understanding radiatively induced Lorentz CPT violation in differential regularization, Phys. Rev. D 60 (1999) 085007 [hep-th/9903258] [INSPIRE].

[30] J.M. Chung and P. Oh, Lorentz and CPT violating Chern-Simons term in the derivative expansion of QED, Phys. Rev. D 60 (1999) 067702 [hep-th/9812132] [INSPIRE].

[31] J.M. Chung and B.K. Chung, Induced Lorentz and CPT violating Chern-Simons term in QED: Fock-Schwinger proper time method, Phys. Rev. D 63 (2001) 105015 [hep-th/0101097] [INSPIRE].

[32] C. Adam and F.R. Klinkhamer, Causality and radiatively induced CPT violation, Phys. Lett. B 513 (2001) 245 [hep-th/0105037] [INSPIRE].

[33] G. Bonneau, Regularisation: many recipes, but a unique principle: Ward identities and normalisation conditions. The case of CPT violation in QED, Nucl. Phys. B 593 (2001) 398 [hep-th/0008210] [INSPIRE].

[34] G. Bonneau, Extended QED with CPT violation: clarifying some controversies, Nucl. Phys. B 764 (2007) 83 [hep-th/0611009] [INSPIRE].

[35] M. Pérez-Victoria, Physical (ir)relevance of ambiguities to Lorentz and CPT violation in QED, JHEP 04 (2001) 032 [hep-th/0102021] [INSPIRE].

[36] W.F. Chen, Issues on radiatively induced Lorentz and CPT violation in quantum electrodynamics, hep-th/0106035 [INSPIRE].

[37] Y.A. Sitenko and K.Y. Rulik, On the effective Lagrangian in spinor electrodynamics with added violation of Lorentz and CPT symmetries, Eur. Phys. J. C 28 (2003) 405 [hep-th/0212007] [INSPIRE].

[38] B. Altschul, Gauge invariance and the Pauli-Villars regulator in Lorentz- and CPT-violating electrodynamics, Phys. Rev. D 70 (2004) 101701 [hep-th/0407172] [INSPIRE].

[39] A.P. Baêta Scarpelli, M. Sampaio, M.C. Nemes and B. Hiller, Gauge invariance and the CPT and Lorentz violating induced Chern-Simons-like term in extended QED,

Eur. Phys. J. C 56 (2008) 571 [arXiv:0804.3537] [INSPIRE]. 
[40] F.A. Brito, J.R. Nascimento, E. Passos and A.Y. Petrov, The ambiguity-free four-dimensional Lorentz-breaking Chern-Simons action, Phys. Lett. B 664 (2008) 112 [arXiv: 0709.3090] [INSPIRE].

[41] J. Alfaro, A.A. Andrianov, M. Cambiaso, P. Giacconi and R. Soldati, Bare and induced Lorentz and CPT invariance violations in QED, Int. J. Mod. Phys. A 25 (2010) 3271 [arXiv: 0904.3557] [INSPIRE].

[42] O.M. Del Cima, J.M. Fonseca, D.H.T. Franco and O. Piguet, Lorentz and CPT violation in QED revisited: a missing analysis, Phys. Lett. B 688 (2010) 258 [arXiv:0912.4392] [INSPIRE].

[43] O.M. Del Cima, D.H.T. Franco, A.H. Gomes, J.M. Fonseca and O. Piguet, All orders renormalizability of a Lorentz and CPT violating quantum electrodynamics, Phys. Rev. D 85 (2012) 065023 [arXiv:1202.0009] [inSPIRE].

[44] J.F. Assunção and T. Mariz, Radiatively induced CPT-odd Chern-Simons term in massless QED, Eur. Phys. Lett. 110 (2015) 41002 [arXiv:1505.08156] [INSPIRE].

[45] J.H. Lowenstein, BPHZ renormalization, in Renormalization theory, G. Velo and A.S. Wightman eds., D. Reidel Publ. Co. (1976).

[46] J.H. Lowenstein and B. Schroer, Gauge invariance and Ward identities in a massive vector meson model, Phys. Rev. D 6 (1972) 1553 [InSPIRE].

[47] J.H. Lowenstein and B. Schroer, Comment on the absence of radiative corrections to the anomaly of the axial-vector current, Phys. Rev. D 7 (1973) 1929 [INSPIRE].

[48] N. Nakanishi, Covariant quantization of the electromagnetic field in the Landau gauge, Prog. Theor. Phys. 35 (1966) 1111 [InSPIRE].

[49] B. Lautrup, Canonical quantum electrodynamics in covariant gauges, Kong. Dan. Vid. Sel. Mat. Fys. Med. 35 (1967), no. 11 [InSPIRE].

[50] S. Weinberg, High-energy behavior in quantum field theory, Phys. Rev. 118 (1960) 838 [INSPIRE].

[51] H. Balasin, M. Schweda, M. Stierle and O. Piguet, The cohomology problems of rigid Lorentz transformations in axial gauge theories, Phys. Lett. B 215 (1988) 328 [INSPIRE].

[52] C. Itzykson and J.-B. Zuber, Quantum field theory, Physics Series, McGraw-Hill (1980).

[53] R. Stora, Pedagogical experiments in renormalized perturbation theory, contribution to the Meeting on the Theory of Renormalization and Regularization, Hesselberg Germany (2002).

[54] S.L. Adler, Axial-vector vertex in spinor electrodynamics, Phys. Rev. 177 (1969) 2426 [INSPIRE].

[55] J.S. Bell and R. Jackiw, A PCAC puzzle: $\pi^{0} \rightarrow \gamma \gamma$ in the $\sigma$-model, Nuovo Cim. A 60 (1969) 47 [inSPIRE].

[56] J.S. Schwinger, On gauge invariance and vacuum polarization, Phys. Rev. 82 (1951) 664 [INSPIRE].

[57] S.L. Adler and W.A. Bardeen, Absence of higher order corrections in the anomalous axial vector divergence equation, Phys. Rev. 182 (1969) 1517 [INSPIRE].

[58] A. Zee, Axial vector anomalies and the scaling property of field theory, Phys. Rev. Lett. 29 (1972) 1198 [inSPIRE].

[59] A. Blasi, O. Piguet and S.P. Sorella, Landau gauge and finiteness, Nucl. Phys. B 356 (1991) 154 [InSPIRE]. 